\title{
LOCAL WAVELET-BASED SPECTRAL "EPSILON" MODIFICATION OF GROUND MOTIONS IN SUPPORT OF INCREMENTAL DYNAMIC ANALYSIS
}

\author{
A. Giaralis ${ }^{1}$ and D. Vamvatsikos ${ }^{2}$ \\ ${ }^{1}$ Department of Civil Engineering, City University London, Northampton Square, \\ EC1V 0HB, London, United Kingdom; email: agathoklis@city.ac.uk \\ ${ }^{2}$ School of Civil Engineering, National Technical University of Athens, Heroon \\ Polytechneioy 9, Athens 157 80, Greece; email: divamva@central.ntua.gr
}

\begin{abstract}
A novel scaling algorithm for ground motion accelerograms (GMs) is proposed in support of incremental dynamic analysis used to establish dependable statistical relationships between scalable intensity measures (IMs) and engineering demand parameters (EDPs) within a performance based earthquake engineering framework. Specifically, an iterative harmonic wavelet based scheme is employed to accomplish "surgical" changes to the spectral shape of suites of GMs to span various pre-defined levels of the spectral acceleration at the structural fundamental natural period, the most widely adopted IM. Since the target IM values may not be accomplished precisely by the local spectral modifications, a second step involving global uniform GM scaling is further considered. The proposed algorithm requires significantly smaller global (amplitude) scaling factors compared to the currently used scaling approach in which no initial local GM modification is undertaken. A numerical application of the proposed algorithm to elastoplastic structural systems shows the extent to which spectral shape may influence the displacement response of yielding structures and explains the conservative bias introduced by uniform global scaling.
\end{abstract}

\section{INTRODUCTION}

The Performance-Based Earthquake Engineering (PBEE) framework requires the dependable knowledge of the mean annual frequency that a specified level of a structural demand quantity will be exceeded or that a certain damage limit-state capacity will be reached for a given structure located at a certain site. To accommodate this requirement, Incremental Dynamic Analysis (IDA, Vamvatsikos and Cornell 2002) is commonly employed to map the response of seismically excited structures from the linear range to strongly nonlinear behavior and, if so desired, to global dynamic instability (collapse). In particular, IDA generates plots (IDA curves) of the peak of a judicially chosen structural response quantity (engineering demand parameter-EDP) versus a gradually increasing seismic intensity measure (IM). This is accomplished by performing a series of response history analyses of a non-linear Finite Element structural model under a judicially selected suite of amplitude-scaled 
strong ground motions (GMs). The employed incremental amplitude scaling with increasing values of the IM (IM-stripes) is assumed to make the considered suite of GMs to become consistent with a range of earthquake scenarios corresponding to different intensities. From an engineering seismology viewpoint, these intensities are commonly defined in terms of the earthquake magnitude $M$, the epicentral distance $R$, and the epsilon parameter $\varepsilon$. Epsilon equals the number of standard deviations by which the logarithm of the spectral acceleration $S_{a}\left(T_{1}\right)$ of a recorded GM evaluated at the fundamental natural period $T_{1}$ of a structure differs from the median logarithm of $S_{a}\left(T_{1}\right)$ calculated from a given attenuation relationship (Baker and Cornell 2005). It can be construed as a measure of the "spectral shape" of a GM close to $T_{1}$ and, thus, as a parameter associated with the frequency content of the GM.

The practice of uniform (global) amplitude scaling of GMs has received criticism in the literature both from a theoretical (Grigoriu 2011) and from a practical viewpoint (Luco and Bazzurro 2007): it raises the question as to how much a recorded GM can be scaled and still be considered as "realistic" and representative of an underlying $(M, R, \varepsilon)$ earthquake scenario. However, this practice becomes a necessity especially for the reliable collapse assessment of code-compliant structures that require the consideration of extremely rare seismic events. For such events insufficient numbers of recorded GMs exist to properly account for the record-torecord structural response variability. In this regard, significant research effort has been devoted to achieve reductions in the bias and variance of various EDPs of practical interest for the high IM levels (stripes) that are required to drive modern earthquake resistant structures to the near collapse state. Specifically, it has been established that a viable way to achieve such reductions involves adopting IMs which should carry as much information as possible, not only for the input seismic action, but also for the considered structure itself (Baker and Cornell 2005). To this aim, single parameter IMs, such as the peak inelastic displacement (Tothong and Luco 2007), and two-parameter (vector-valued) IMs, such as $S_{a}\left(T_{1}\right)$ and $\varepsilon\left(T_{1}\right)$ (Baker and Cornell 2005), have been proposed in the literature. However, the most commonly used IM among engineers remains to be the $S_{a}\left(T_{1}\right)$ since it is widely adopted in defining seismic hazard maps and attenuation relationships and is also used by current codes of practice to define the seismic input action. An alternative way to reduce the bias and variance in estimating peak inelastic structural response demands is through a careful selection of the suite of GMs considered which may involve individual scaling to meet certain compatibility criteria with the $(M, R, \varepsilon)$ earthquake scenario (Baker and Cornell 2006). Despite the recent advances in record selection algorithms (e.g. Katsanos and Sextos 2013), selection and perhaps replacement of GMs as the IM level increases are a rather algorithmic-dependent step, let alone computationally demanding.

To circumvent some of the above limitations of the current state of art in achieving IM sufficiency, this paper explores the potential of scaling earthquakes in a non-uniform (local) manner within a narrow range of periods centered at the fundamental natural period $T_{1}$, or, equivalently, within a narrow band of frequencies centered at the fundamental natural frequency of a structure. This is efficiently accomplished by using an iterative harmonic wavelet-based scheme whose potential to produce spectral matched accelerograms within arbitrarily defined frequency bands 
is well-established in the literature (Giaralis and Spanos 2009, 2010). Specifically, a novel algorithm is proposed according to which harmonic wavelets are initially used to accomplish "surgical" changes to the spectral shape ("epsilon") of a selected suite of accelerograms to span various pre-defined IM-stripes expressed by "target" $S_{a}\left(T_{1}\right)$ values prior to global (uniform) scaling.

In what follows, the adopted harmonic wavelet based spectral modification procedure is briefly reviewed. Representative numerical data are reported and discussed on its effectiveness to achieve local spectral scaling at different prespecified IM-stripes. Next, the proposed novel algorithm is presented and applied to two different yielding structural systems considering a suite of GMs used in the FEMA P-695 report (FEMA 2009). Summarized IDA curves are obtained from IDA analyses using only uniformly scaled records (the conventional way) and using locally modified records (the herein proposed algorithm) and compared. The paper concludes with a critical discussion on the reported numerical data pointing to future research directions.

\section{HARMONIC WAVELET BASED MODIFICATION OF ACCELEROGRAMS}

Introduced by Newland (1994), the harmonic wavelet transform (HWT) has been proved to yield useful time-frequency representations of non-stationary signals encountered in the field of earthquake engineering and structural damage detection (e.g. Spanos et al. 2007). The HWT decomposes a given signal onto a basis of complex-valued analyzing functions, termed "harmonic wavelets", which attain a band-limited Fourier spectrum in the domain of frequencies $\omega$ and observe a decaying oscillatory waveform with time $t$. A "general" harmonic wavelet $\psi_{(m, n), k}$ of $(m, n)$ scale centered at the $k /(n-m)$ position in time is defined as a function possessing a constant spectrum within the frequency band $[m 2 \pi, n 2 \pi)$, where $k, m$, and $n$ are real positive numbers (Newland 1994). The Fourier transform of this wavelet reads as

$$
\hat{\Psi}_{(m, n), k}(\omega)=\left\{\begin{array}{cl}
\frac{1}{(n-m) 2 \pi} \exp \left(\frac{-i \omega k}{n-m}\right), & m 2 \pi \leq \omega<n 2 \pi \\
0 \quad, & \text { otherwise }
\end{array} .\right.
$$

Consider a collection of harmonic wavelet functions spanning adjacent nonoverlapping bands of arbitrary widths at different scales along the whole frequency axis. At each scale $\left(m_{j}, n_{j}\right)$, the considered collection encompasses wavelets centered at all possible $k /\left(n_{j}-m_{j}\right)$ time instants. Newland (1994) has shown that such a collection constitutes a complete and orthogonal basis for finite energy real signals $f(t)$. By utilizing this basis, the HWT expressed by

$$
W(m, n, k)=(n-m) \int_{-\infty}^{\infty} f(t) \overline{\psi_{(m, n), k}(t)} d t,
$$

where the bar over a symbol denotes complex conjugation, yields a signal representation which preserves the signal energy $E$. This can be mathematically expressed by the equation (Giaralis and Spanos 2010) 


$$
E=\int_{-\infty}^{\infty} f(t)^{2} d t=2 \sum_{m, n} \frac{1}{n-m} \sum_{k}\left|W_{(m, n), k}\right|^{2},
$$

where the summation over $m, n$ accounts for all $(m, n)$ scales considered and the summation over $k$ accounts for all wavelets located at $k /\left(n_{j}-m_{j}\right)$ in time. Therefore, it can be deduced that the HWT decomposes the signal $f(t)$ into band-limited subsignals $f_{m, n}(t)$ expressed by (Spanos et al. 2005)

$$
f_{m, n}(t)=2 \operatorname{Re}\left\{\sum_{k} W_{(m, n), k} \psi_{(m, n), k}(t)\right\} .
$$

In light of Eq. (3), it can be readily shown that the original accelerogram $f(t)$ can be reconstructed by summing up the above sub-signals. That is,

$$
f(t)=\sum_{m, n} f_{m, n}(t)
$$

Note that the energy of each $f_{m, n}(t)$ component is concentrated within the $[m 2 \pi, n 2 \pi)$ interval in the frequency domain. Consequently, Giaralis and Spanos (2009) noted that the response of a lightly damped linear oscillator of natural period $T$ lying within the interval $\left(1 / n_{j}, 1 / m_{j}\right]$ base-excited by an acceleration time-history $f(t)$ would be mainly influenced by the $f_{m j, n j}(t)$ component. Thus, an iterative modification procedure can be devised to scale all sub-signals at the $v$-th iteration according to the equation (Giaralis and Spanos 2009, Giaralis and Spanos 2010)

$$
f_{m, n}^{(v+1)}(t)=f_{m, n}^{(v)}(t) \frac{\int_{1 / n}^{1 / m} S_{\text {target }}(T) d T}{\int_{1 / n}^{1 / m} S^{(v)}(T) d T},
$$

to yield a modified accelerogram with a response spectrum matching any given response (target) spectrum $S_{\text {target }}(T)$. In Eq. $(6), S^{(v)}(T)$ is the response spectrum of the $f^{(v)}(t)$ obtained by Eq. (5) at each iteration.

It is noted parenthetically, that in the above development continuous-time signals of infinite length have been assumed. However, in practice, band-limited discrete-time finite-duration recorded accelerograms sampled at a constant time interval are to be processed. To this aim, a fast Fourier transform based algorithm can be employed for the efficient computation of the convolution integral of Eq. (2) in the frequency domain (Newland 1999). Furthermore, a sufficient number of scales/"bins" must be considered to cover all frequencies of interest. A detailed discussion along with numerical evidence on the impact of the width of these bins on the efficiency of the above described iterative procedure can be found in Giaralis and Spanos (2009).

\section{LOCAL SPECTRAL MODIFICATION OF ACCELEROGRAMS}

For the purpose of this study, the aforementioned harmonic wavelet based local spectral modification procedure is used to modify ground motion acceleration records 
(GMs) locally, within a pre-specified narrow range of periods centered at $T=T_{I}$ period, such that they span any desired "IM-stripe" as required by the standard Incremental Dynamic Analysis (IDA) (Vamvatsikos and Cornell 2002). To illustrate the procedure and the effectiveness of harmonic wavelets to achieve the desired modification, representative results corresponding to a particular GM recorded during the Northridge 1994 earthquake (Beverly Hills-14145 MULH-009 component) are reported in Figure 1.
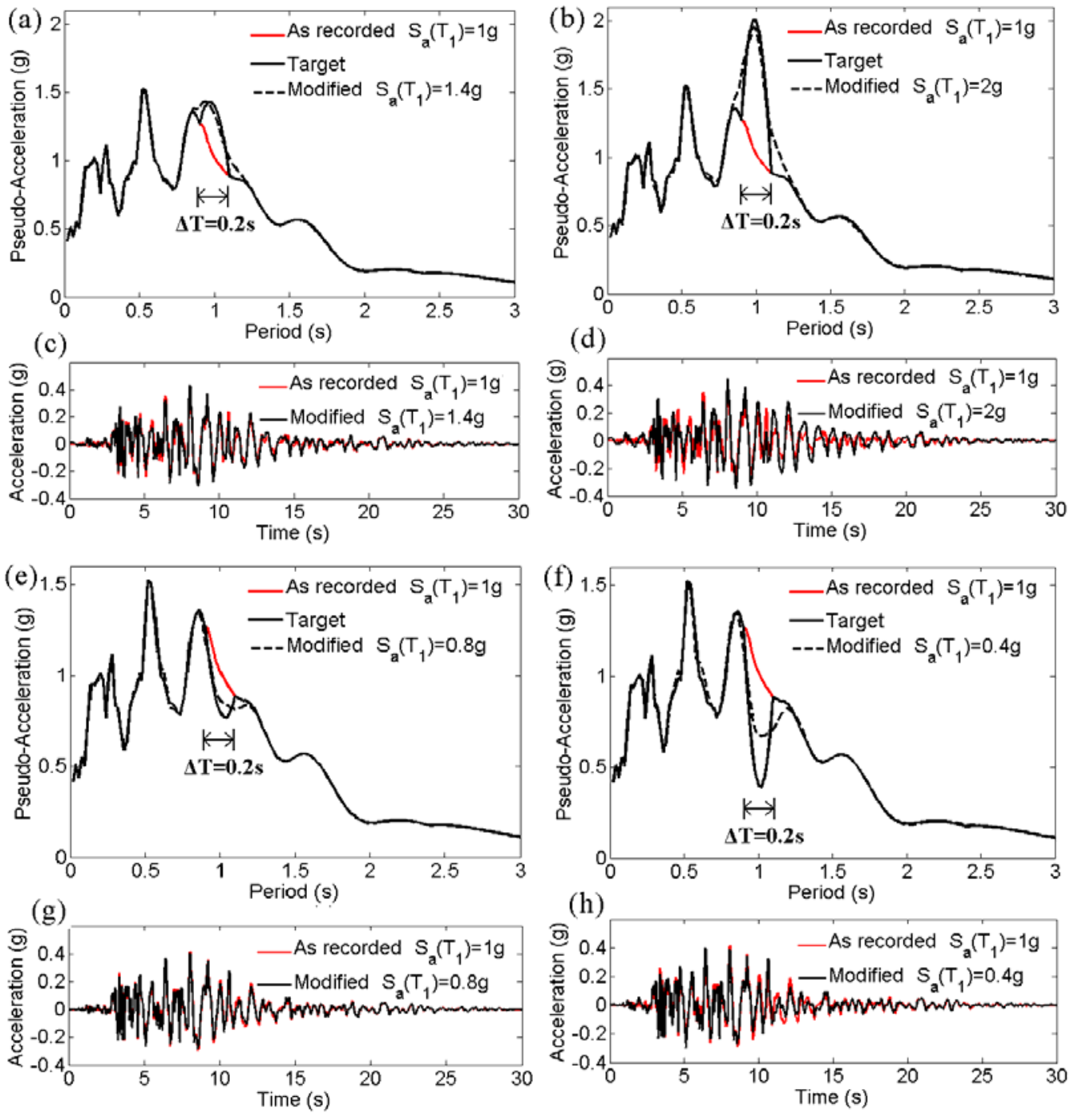

Figure 1. Local harmonic wavelet (epsilon) modification within a period range of $\Delta T=0.2 \mathrm{~s}$ centered at $T_{1}=1 \mathrm{~s}$ of the Beverly Hills-14145 MULH-009 component (Northridge 1994 earthquake) to achieve $S_{a}\left(T_{1}=1 \mathrm{~s}\right)=0.4 \mathrm{~g}, 0.8 \mathrm{~g}, 1.4 \mathrm{~g}$, and $2 \mathrm{~g}$.

Specifically, it is assumed that a structure with $T_{l}=1 \mathrm{~s}$ fundamental natural period is of interest. This particular ("as recorded") GM observes a spectral acceleration value $S_{a}^{\text {rec }}\left(T_{1}\right)=1 g$, where $g$ is the gravitational acceleration. Local harmonic wavelet based modification (non-uniform scaling) of the above signal is 
undertaken to obtain different accelerograms corresponding to four specific $S_{a}\left(T_{1}\right)$ values (or IM-stripes), namely $0.4 \mathrm{~g}, 0.8 \mathrm{~g}, 1.6 \mathrm{~g}$ and $2 \mathrm{~g}$. This has been achieved in each case by considering as an initial (seed) signal in the iterative procedure represented by Eq. (6) the "as recorded" GM. As "target" spectrum, $S_{\text {target }}(T)$, the response spectrum of the "as recorded" signal is taken scaled (up or down) within a fixed interval $\left(0.9 T_{1}, 1.1 T_{1}\right)=(0.9,1.1) \mathrm{s}$ of $\Delta T=0.2 \mathrm{~s}$ width such that $S_{\text {target }}\left(T_{1}\right)$ becomes equal to each of the four desired $S_{a}\left(T_{1}\right)$ values, as shown in panels (a),(b),(e) and (f) of Figure 1. These smooth locally up/down scaled target spectra have been derived by cubic spline interpolation such that they coincide exactly with the response spectrum of the "as recorded" GM outside the interval $\left(0.9 T_{1}, 1.1 T_{1}\right)$, that is $S_{\text {target }}(T)=S_{a}^{\text {rec }}(T)$ for all $T \neq\left(0.9 T_{1}, 1.1 T_{1}\right)$, while $S_{\text {target }}\left(T_{l}\right)=S_{a}\left(T_{1}\right)$. The response spectra and timehistory traces of the four locally modified accelerograms are plotted in Figure 1 vis-àvis those corresponding to the considered "as recorded" accelerogram. These modified accelerograms have been obtained by considering a basis of harmonic wavelets spanning non-overlapping frequency bands of $\Delta \omega_{c}=0.0383 \mathrm{rad} / \mathrm{s}$ width within the "critical" interval $\left(2 \pi / 1.1 T_{1}, 2 \pi / 0.9 T_{1}\right)=(5.712,6.981) \mathrm{rad} / \mathrm{s}$ where local up/down scaling is enforced and of $\Delta \omega=\Delta \omega_{c} / 16=0.6128 \mathrm{rad} / \mathrm{s}$ width outside this interval. Six iterations have been performed.
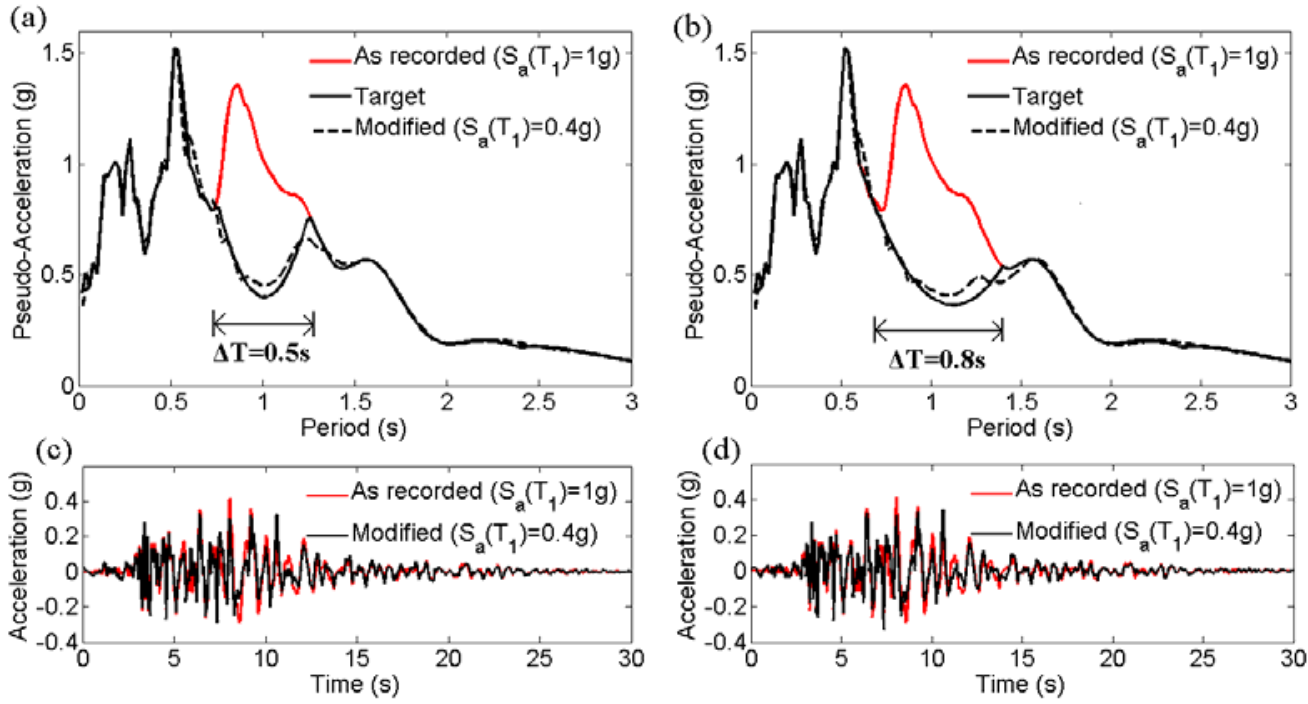

Figure 2. Local harmonic wavelet (epsilon) modification within two different period ranges $\Delta T$ centered at $T_{1}=1 \mathrm{~s}$ of the Beverly Hills-14145 MULH-009 component (Northridge 1994 earthquake) to achieve $S_{a}\left(T_{1}=1 \mathrm{~s}\right)=0.4 \mathrm{~g}$.

The reported results of Figure 1 are representative of the quality of spectral matching achieved by the harmonic wavelet for a number of GMs tested by the authors. In particular, it is seen that the adopted iterative harmonic wavelet scheme obtains accelerograms with spectral values very close to $S_{a}\left(T_{1}\right)$ values typically desired in IDA analysis when $S_{a}\left(T_{1}\right)>S_{a}^{\text {rec }}\left(T_{1}\right)$ (i.e. local scaling up) without changing the response spectrum shape outside the specified interval about $T_{1}$ and, more importantly, without significant changes to the seed "as recorded" GM (see also 
Giaralis and Spanos 2010 for further discussion). However, when local scaling down is required (i.e. $S_{a}\left(T_{1}\right)<S_{a}^{r e c}\left(T_{1}\right)$ ), the algorithm does not perform as well as in the case of local scaling up. This is due to the fact that non-negligible frequency content of the input signal lying close to (but not coinciding to) the natural frequency $2 \pi / T_{l}$ of a linear oscillator has a non-negligible influence to the peak response of this oscillator. A reasonable way to rectify this issue is to widen the "critical" interval around $T_{1}$. Figure 2 includes pertinent results for two different $\Delta \mathrm{T}$ widths of the interval centered at $T_{1}$ for the same recorded accelerogram to numerically exemplify this issue. Nevertheless, it is noted that from a practical viewpoint the local scaledown is not of particular interest as it is likely that sufficient number of recorded GMs corresponding to low IM values exist in well populated data bases. The practical merit of the proposed algorithm utilizing the above harmonic wavelet based local modification described in the next section aims to circumvent primarily the lack of a sufficient number of recorded GMs corresponding to large IM values and, therefore, to rare large intensity seismic events.

\section{PROPOSED SCALING ALGORITHM USING LOCAL SPECTRALLY (“EPSILON") MODIFIED ACCELEROGRAMS IN SUPPORT OF IDA}

Herein, a novel scaling algorithm for incremental dynamic analysis (IDA) is proposed aiming to derive accelerograms corresponding to high IM values without application of unrealistically large global scaling factors to as recorded GMs. The algorithm resembles the classical stepping algorithm of IDA (Vamvatsikos and Cornell 2002) and exploits the capability of the previously discussed iterative harmonic wavelet based modification scheme to accomplish "surgical" local modifications to the response spectra of recorded GMs prior to application of (uniform) global scaling according to the scalable $S a\left(T_{1}\right)$ IM. The steps of the proposed algorithm applied to a predefined suite of strong ground motion records are summarized as follows

Set $S_{\text {step }}$ and $\Delta \mathrm{T}$

For each record

$$
\begin{aligned}
& S_{\text {target }}\left(T_{1}\right)=0 \\
& \text { Repeat } \\
& S_{\text {target }}\left(T_{1}\right)=S_{\text {target }}\left(T_{1}\right)+S_{\text {astep }} \\
& \text { Modify locally record within } \Delta \mathrm{T} \text { such that } S\left(T_{1}\right) \approx S_{\text {target }}\left(T_{1}\right) \\
& \text { Compute spectral value } S_{\text {actual }}\left(T_{1}\right) \text { of modified record } \\
& \text { Scale uniformly modified record by factor } S_{\text {target }}\left(T_{1}\right) / S_{\text {actual }}\left(T_{1}\right) \\
& \text { Run dynamic analysis }
\end{aligned}
$$

\section{End for}

According to the above algorithm, uniform (amplitude) scaling of GMs is only applied after "local modification" takes place within a predefined (typically narrow) range of periods $\Delta \mathrm{T}$ centered at the structural fundamental period $T_{1}$ of interest to take care of any (typically small) inaccuracy in achieving the 
$S_{\text {target }}\left(T_{1}\right)$ value (see also Figure 1). Thus, the algorithm keeps the requirement for global (uniform) up/down scaling of GMs, whose use is currently under debate (e.g. Luco and Bazzuro 2007, Grigoriu 2011), to a minimum. It is noted in passing that the herein considered harmonic wavelet-based "local modification" achieves a change of the epsilon $\varepsilon\left(\mathrm{T}_{1}\right)$ of each record prior to global uniform scaling (see e.g. Baker and Cornell 2005). Further intuition can be gained by interpreting the suite of records as samples of an underlying stochastic process. Under this assumption, the initial local scaling step is equivalent to adding/subtracting a narrow band-like stochastic process with central frequency approximately equal to $2 \pi / T_{1}$ while the subsequent global scaling is equivalent to adding/subtracting a white noise-like stochastic process.

\section{NUMERICAL APPLICATION OF PROPOSED ALGORITHM}

For application, a simple elasto-plastic oscillator with a period of $T_{1}=1 \mathrm{~s}$ is utilized. Since such a system does not experience collapse, a limiting displacement, or ductility, is considered to introduce dynamic instability. Two different oscillators are employed, one with a limiting ductility of $\mu_{u}=2.5$, representative of a moderate ductility system, and another that can achieve higher ductility with $\mu_{u}=4.0$. Each system is subjected to classical IDA (global uniform scaling only) and to the novel algorithm described in the previous section (local wavelet-based modification prior to global scaling). An ensemble of 44 GMs comprising the far-field GM databank used in FEMA P-695 report (FEMA 2009) have been considered.

A comparison of the results for the two aforementioned systems appears in Figure 3. For the moderately ductile system (left panel of Figure 3), the two different scaling strategies considered produce essentially the same results. Some minor differences can be detected but they are statistically insignificant given the size of the record set. Quite the opposite happens for the second system, whose higher ductility capacity allow the effect of spectral shape to appear (right panel of Figure 3). Apparently, the more "spiky" spectral shape of the locally modified records leaves them weak in the longer period range. Thus, when the "apparent" natural period of the oscillator shifts towards longer periods (see e.g. Katsanos et al. 2013), the spectrally modified records cannot readily drive the system to higher response. Nevertheless, this effect does not appear uniformly over all the GMs considered. The $16 \%$ curves of response are practically the same for the two scaling methods, meaning that the most aggressive of GMs are not affected much by the scaling strategy adopted, at least not for ductility lower than 4.0. It may be postulated that these might be the ones that showed relatively strong long-period content, although a more detailed investigation should be carried out to confirm this. The median curve estimated by IDA stays on an equal displacement trajectory for this moderate period system: This is exactly what is predicted by most strength reduction factor / ductility / period $(R-\mu-T)$ relationships. Still, the locally modified ground motions deviate from this canon when ductility higher than 3 is reached. The most dramatic changes are shown by the most benign records, as presented by the $84 \%$ curve. Signs of "saturation" are exhibited for intensities between $0.6 \mathrm{~g}$ and $1.2 \mathrm{~g}$, showing a slowly increasing response that only accelerates closer to final collapse. 
Naturally, one should not argue in favor of one method against the other. It is actually quite probable that both are equally wrong, as the best overall method would be the one that would perfectly reproduce the seismologically correct spectral shape (and its statistical properties, e.g., central value and variability) that should be expected at each intensity level. This could potentially be the conditional spectrum suggested by Baker and Cornell (2006) or some more elaborate version that is derived by seismological simulations to be fully consistent with the site characteristics (e.g. Vetter and Taflanidis 2012). Higher intensities may actually mean differences in the spectral shape depending on the site itself. For example, a high $S_{a}\left(T_{1}\right)$ value for a relatively small fault may be caused by the generation of a nearsource pulse for a moderate magnitude event, while the same may be achieved by a more distant fault for a larger magnitude event without any directivity effect appearing in the site of interest. The first process may be associated with a more "spiky" spectral shape, while the latter may produce a more broadband GM.

In other words, it is quite probable that the truth may be somewhere between the two considered scaling procedures and it may be best reached by a combination of local modification and global scaling, the amount of each to be determined by the actual site being studied. Still, having the two methods overlaid in the same graph can be a useful tool to understand the limitations of both and perhaps even set some bounds on what response can be expected from a given set of GMs.
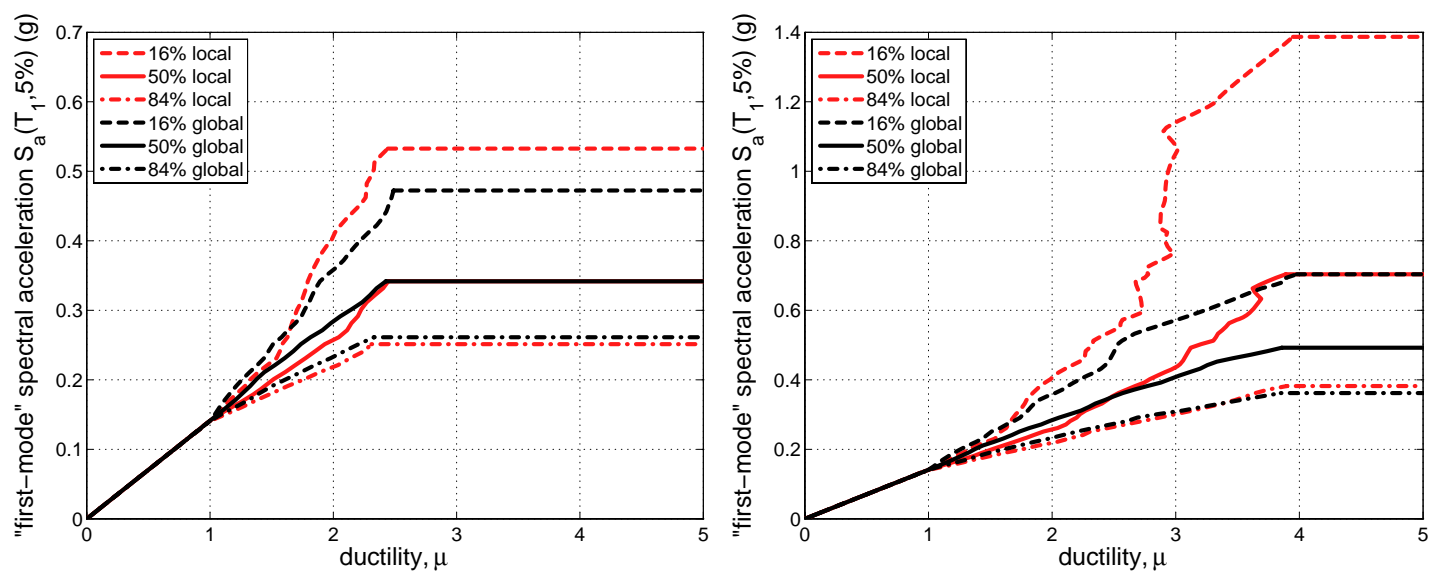

Figure 3. 16/50/84\% fractile IDA curves comparing the local (wavelet) versus the global (scaling) modification for an elastoplastic system with $T=1 \mathrm{sec}$ and an ultimate ductility of (a) $\mu_{u}=2.5$, (b) $\mu_{u}=4$.

\section{CONCLUSIONS}

A novel algorithm in support of incremental dynamic analysis has been introduced involving local harmonic wavelet based spectral (epsilon) modification of recorded ground motion accelerograms (GMs) to achieve predefined IM levels in terms of spectral acceleration $S_{a}\left(T_{1}\right)$ values without the need to use unrealistically large global (uniform) scaling factors. Application to simple elasto-plastic oscillators shows that for moderate period systems experiencing low-to-moderate levels of ductility (of the order of 3), both the proposed novel scaling procedure and the classical (global) 
scaling one produce practically the same results. When higher levels of ductility are reached, though, the displacement response of the oscillator rapidly saturates for locally modified records, an effect indicative of their weaker spectral content outside the relatively narrow period range within which local scaling is sought. Arguably, this phenomenon can be related to the so-called apparent "period elongation" which becomes significant for near-collapse hysteretic structures. In any case, taking a very narrow period range around $T_{1}$ to scale GMs as applied herein lies at the opposite end of global scaling in terms of the extent of GM modification in the frequency domain. To this end, the final conclusion drawn based on the so-far numerical data available to the authors is that there is a trade-off as to how much local versus global scaling should be pursued. Further research work is underway to shed light to the above trade-off and to determine practically useful recommendations on this issue based on IDA results (i.e. statistics of the peak response of inelastic structural systems).

\section{REFERENCES}

Baker, J. W., and Cornell, C. A. (2005). “A vector-valued ground motion intensity measure consisting of spectral acceleration and epsilon." Earthq. Eng. Struct. Dyn., 34, 1193-1217.

Baker, J. W., and Cornell C. A. (2006). "Spectral shape, epsilon and record selection." Earthq. Eng. Struct. Dyn., 35, 1077-1095.

Federal Emergency Management Agency (2009). "Recommended Methodology for Quantification of Building System Performance and Response Parameters," FEMA P-695, prepared by the Applied Technology Council, Redwood City, CA.

Giaralis, A., and Spanos, P. D. (2009). "Wavelet-based response spectrum compatible synthesis of accelerograms-Eurocode application (EC8).” Soil Dyn. Earthq. Eng., 29, 219-235.

Giaralis, A., and Spanos P. D. (2010). "Derivation of Eurocode 8 spectrum-compatible time-histories from recorded seismic accelerograms via harmonic wavelets." In: Proceedings of the $9^{\text {th }}$ HSTAM International Congress on Mechanics,(eds: Papanastasiou, P., Roussis, P., Loukidis, D., Sarris, E. and Katsikadelis, J.), paper \#023, pp. 669-677.

Grigoriu, M. (2011). "To scale or not to scale seismic ground acceleration records." J. Eng. Mech., ASCE, 137, 284-293.

Katsanos, E. I., Sextos, A.G., and Elnashai A.S. (2013). "Period elongation of nonlinear systems modeled with degrading hysteretic rules." In: $15^{\text {th }}$ World Conference in Earthquake Engineering, Lisbon.

Katsanos, E. I., and Sextos, A.G. (2013). "ISSARS: An integrated software environment for structure specific earthquake ground motion selection." Advances Eng. Software, 58, 70-85.

Luco, N., and Bazzurro, P. (2007). "Does amplitude scaling of ground motion records result in biased nonlinear structural drift responses?” Earthq. Eng. Struct. Dyn., 36(13), 1813-1835.

Newland, D.E. (1994). "Harmonic and musical wavelets," Proc. Royal Soc. London A 444, 605-620.

Newland, D.E. (1999). "Ridge and phase identification in the frequency analysis of transient signals by harmonic wavelets." J. Vibr. Acoust., 121, 149-155.

Tothong, P., and Luco, N. (2007). "Probabilistic seismic demand analysis using advanced ground motion intensity measures." Earthq. Eng. Struct. Dyn., 36, 1837-1860.

Vamvatsikos, D., and Cornell, C. A. (2002). "Incremental Dynamic Analysis." Earthq. Eng. Struct. Dyn., 31, 491-514.

Spanos, P.D., Tezcan, J. and Tratskas, P. (2005). "Stochastic processes evolutionary spectrum estimation via harmonic wavelets," Comp. Meth. App. Mech. Eng., 194, 1367-1383.

Spanos, P.D., Giaralis, A., Politis, N.P. and Roessett, J.M. (2007). "Numerical treatment of seismic accelerograms and of inelastic seismic structural responses using harmonic wavelets," Computer-Aided Civil Infrastruct. Eng., 22, 254-264.

Vetter, C. and Taflanidis, A.A. (2012). "Probabilistic sensitivity analysis for stochastic ground motion modeling in seismic risk assessment," Soil Dyn. Earthq. Eng., 38, 128-143. 